\title{
Fungal Osteomyelitis of Temporomandibular Joint and Skull Base Caused by Chronic Otitis Media
}

\author{
Bok Eum Kim, Keun Jeong Park, Jung Eun Lee, YounJung Park, Jeong-Seung Kwon, \\ Seong-Taek Kim, Jong-Hoon Choi, Hyung-Joon Ahn
}

Department of Orofacial Pain and Oral Medicine, Dental Hospital, Yonsei University College of Dentistry, Seoul, Korea

Received December 18, 2019

Revised February 19, 2020

Accepted February 19, 2020

\section{Correspondence to:}

Hyung-Joon Ahn

Department of Orofacial Pain and Oral

Medicine, Dental Hospital, Yonsei University

College of Dentistry, 50-1 Yonsei-ro,

Seodaemun-gu, Seoul 03722, Korea

Tel: +82-2-2228-3113

Fax: +82-2-393-5673

E-mail: HJAHN@yuhs.ac

https://orcid.org/0000-0001-9669-9781

This work was supported by the National Research Foundation of Korea (NRF) grant funded by the Korea government (MIST) (No. 2016R1A5A2008630).

\begin{abstract}
Chronic otitis media (COM) is a chronic inflammatory disease which affects the middle ear, mastoid cavity. It presents hearing loss, ear pain, dizziness, headache, temporomandibular joint (TMJ) inflammation and intracranial complication. Intracranial complications such as skull base osteomyelitis (SBO) may occur secondary to COM due to transmission of infection by a number of possible routes. SBO is an uncommon condition with a significant morbidity and mortality if not treated in the early stages. We report a-67-year-old male patient with diabetes and untreated COM who presented atypical severe TMJ, periorbital and postmandibular pain. By computerized tomography (CT), magnetic resonance imaging (MRI) and whole body bone scan (WBBS), he was diagnosed with SBO spreading from untreated COM via infective arthritis of TMJ. Through this case, we suggest proper utilization of diagnostic imaging, especially CT or MRI for the early detection of SBO in the case of COM accompanying with the greater risk of infection developments such as diabetes.
\end{abstract}

Key Words: Diabetes mellitus; Infection; Osteoarthritis; Osteomyelitis; Otitis media

\section{INTRODUCTION}

Chronic otitis media (COM) is a chronic inflammatory disease of the middle ear and mastoid cavity that consists of a set of interrelated clinical phenotypes. The prevalence in adults is rare [1]. In United Kingdom, its prevalence is 1.5\% and $2.6 \%$ for active and inactive disease respectively [2].

The symptoms of COM are hearing loss, ear pain, dizziness and headache. Other possible symptoms include intracranial complication and temporomandibular joint (TMJ) inflammation [3,4]. Intracranial complications such as skull base osteomyelitis (SBO) may occur by transmission of infection based on COM [5]. Typical otogenic SBO generally develops in people over 60 with uncontrolled diabetes and immunocompromised host: acquired immune deficiency syndrome and blood proliferative disease. SBO is an uncommon condition with a significant morbidity and mortality if not detected and treated in the early stages [6]. The etiological factor is Pseudomonas aeruginosa in 99.2\% [7]. Fungal SBO is much rarer, where the causative pathogens are often Aspergillus species [6].

In this paper, we present the case of a-67-year-old male patient with diabetes and untreated COM who was diagnosed with SBO.

\section{CASE REPORT}

A-67-year-old man visited the Department of Orofacial Pain and Oral Medicine of Yonsei University Dental Hospital (Seoul, Korea) with a complaint of his left TMJ pain. There has been a history of otorrhea from his left ear 1 month before. After treatment in otolaryngology, the symptom was

Copyright (c) 2020 Korean Academy of Orofacial Pain and Oral Medicine. All rights reserved.

(cc) This is an open-access article distributed under the terms of the Creative Commons Attribution Non-Commercial License (http://creativecommons.org/licenses/by-nc/4.0/), which permits unrestricted non-commercial use, distribution, and reproduction in any medium, provided the original work is properly cited. 
improved. However, there was severe pain in the left TMJ and around the orbit, with trismus about 2 weeks ago prior to his first visit to clinic. He had already taken nonsteroidal anti-inflammatory drugs from local dental clinic, and his symptoms had been relieved but not disappeared. The patient had been taking medication for the treatment of hypertension, diabetes and COM, but not been treated for COM. The TMJ cone beam computerized tomography was taken in other dental clinic 1 week prior to his first visit and showed no abnormal finding (Fig. 1). The patient had a maximum mouth opening of $42 \mathrm{~mm}$ with pain in the left TMJ. There were no restriction of both condylar movement and no palpable tenderness on any masticatory muscles, but he showed familiar pain to palpation in the left TMJ. Occlusal condition was stable. He was diagnosed with TMJ arthralgia in the left TMJ. After medication (Airtal [aceclofenac; Daewoong Co., Seoul, Korea], Mucosta [rebamipide; Otsuka Co., Seoul, Korea]), the pain in the left TMJ was disappeared. He was recommended treatment for COM and discharged because his left intra-auricular pain had been persisted.

A 6 months later, he was referred to our clinic by otolaryngologist and presented with pricking and throbbing pain in the postauricular area. Computed tomography (CT) that was taken in other clinic 3 weeks before visit revealed remarkable condylar resorption and erosive change in the left TMJ (Fig. 2) He was diagnosed with TMJ osteoarthritis and suspected infective arthritis in Left TMJ and prescribed medicines including Imotun (avocado soya unsaponifiables; Chong Kun Dang Co., Seoul, Korea), Moxicle (amoxicillin hydrate; Daewoong Co.), Ketolacin (ketorolac tromethamine; Dongkook Co., Seoul, Korea) and Mucosta (rebamipide). However, his severe intermittent pain had been persisted after 1 week. Careful history taking revealed that the pain in the left peri-orbital, postmandibular, parietal and mandibular body area stated more than 2 months before and he had been recommended for surgery for COM but not undergone surgery because of the fear about surgery. Laboratory tests including complete blood count, routine chemistry and C-reactive protein (CRP) and whole body bone scan (WBBS) were carried out for further evaluation. Laboratory tests showed that the level of CRP is 49.1 (normal range 0-8). WBBS showed increased uptake in the left

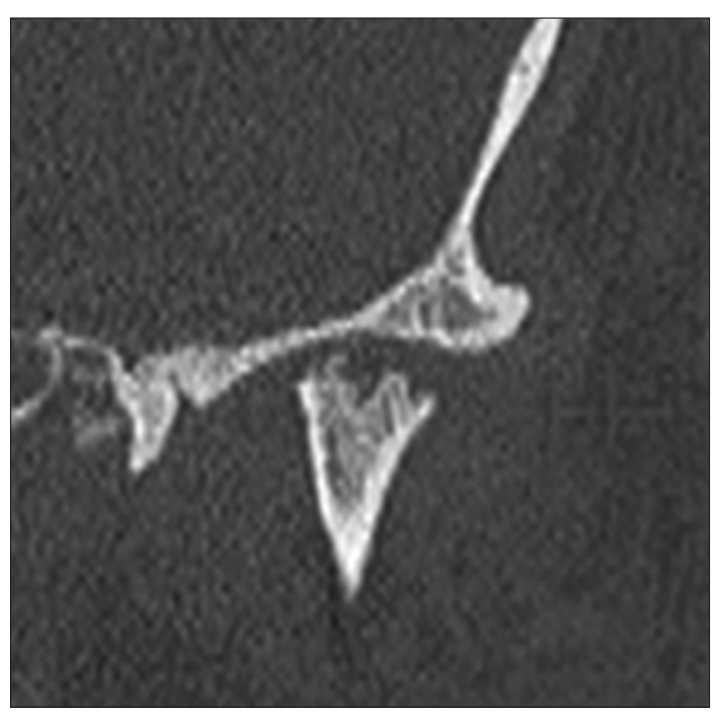

Fig. 2. Computerized tomography taken 6 months after first visit. It revealed severe erosive change on left condyle.
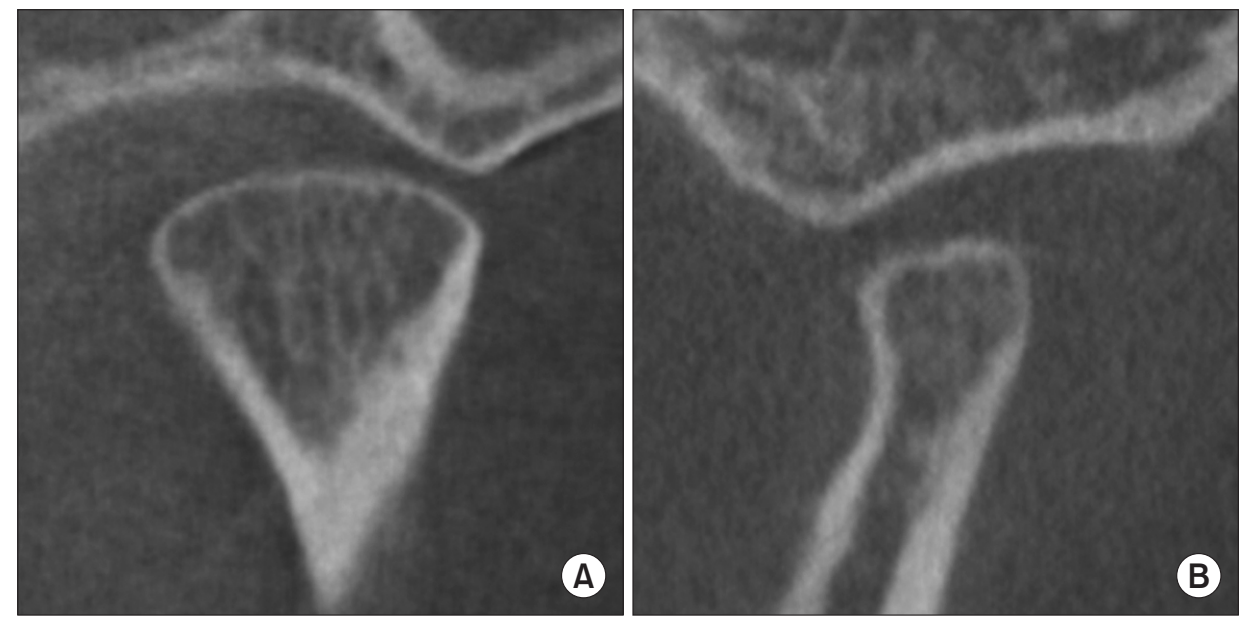

Fig. 1. Cone beam computerized tomography of the left temporomandibular joint taken 1 week prior to first visit. It revealed no remarkable findings. (A) Coronal view. (B) Sagittal view. 

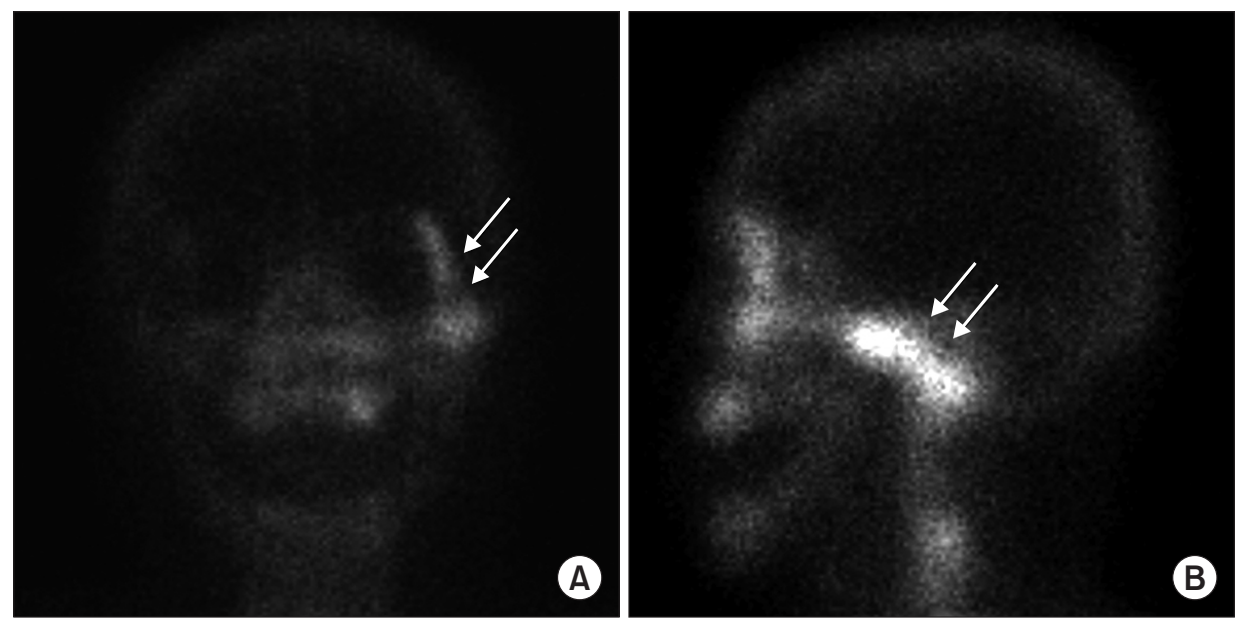

Fig. 3. Whole body bone scan taken 6 months after first visit. It reveals increased uptake (white arrows) in the left zygomatic bone and adjacent orbit. (A) Coronal view. (B) Sagittal view.
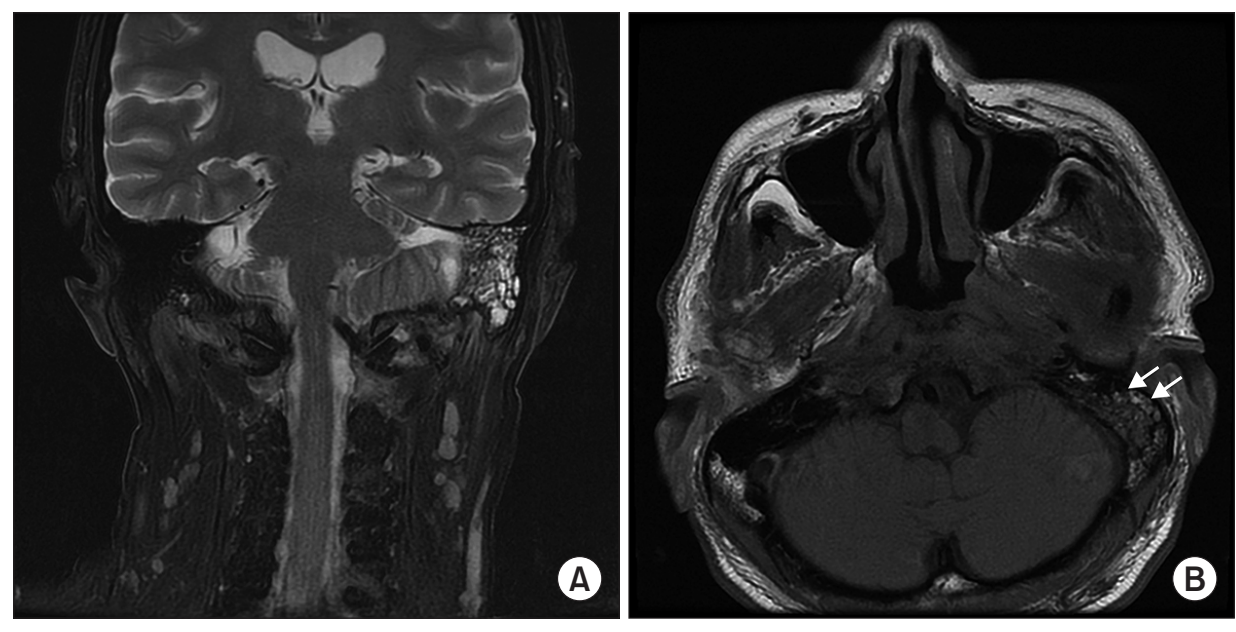

Fig. 4. Face magnetic resonance imaging taken 7 months after first visit. It revealed heterogeneous enhancement (white arrows) in the left condyle and zygomatic bone. (A) Coronal view. (B) Axial view.

zygomatic bone and adjacent orbit (Fig. 3). Magnetic resonance imaging (MRI) scan of face was taken. A MRI scan showed heterogeneous enhancement in the left condyle, zygomatic bone, lateral pterygoid muscle, orbicularis oculi muscle and the anterior margin of the parotid gland (Fig. 4). He was referred to the department of infectious diseases for further evaluation and treatment.

Brain MRI scan was taken and revealed diffuse enhancement of entire posterior skull base. Therefore, left mastoidectomy was performed in the department of otolaryngology and left TMJ arthrocentesis was performed in the department of oromaxillofacial surgery. When the microbial cultures of the surgically resected specimens were performed, candida was found, which finally led to the diagnosis of fungal osteomyelitis of skull base.

\section{DISCUSSION}

COM is a chronic inflammatory disease of the middle ear, mastoid cavity. This is highly prevalent disease and lead to significant morbidity worldwide. However, its aetiology, initiation and perpetuation remain unclear [1]. A symptom of COM is hearing loss, mild to severe ear pain, dizziness and headache. Infections in the mastoid space can cause swelling and pain behind the ear. Other possible symptoms include intracranial complication, TMJ inflammation. Intracranial complications such as SBO, bacterial meningitis, brain abscess, dural sinus thrombosis may occur secondary to COM due to transmission of infection by a number of possible routes $[5,8]$.

This is a case of fungal osteomyelitis of skull base caused by COM. SBO is a rare and can lead to death if not treated 
early. It is most common in elderly persons with diabetes and immunocompromised patients $[9,10]$. It presents in different forms: otogenic and non-otogenic [6,11]. Most otogenic SBO is mainly caused by $P$. aeruginosa $[7,12]$. $P$. aeruginosa infection causes headaches, ear pain, facial pain, swelling around the orbital area. SBO can also be caused by fungus, which is a rarer case [7,13]. It may appear in the ear canal or middle ear in the form of acute, COM, which can also cause chronic maxillary sinusitis, pain in the maxillary sinus, and facial, periorbital swelling $[6,14,15]$.

SBO is a life-threatening disease if not treated properly. Therefore, when dealing with SBO, it is necessary to clarify whether the cause of SBO is fungus or bacteria. If cause of SBO is bacteria, the general treatment consists of long-term antimicrobial treatment with surgical intervention [6]. If cause of SBO is fungus, it can be treated with administration of anti-fungal agents and aggressive surgical debridement [7]. In case of complaining pain on TMJ, but showing atypical pain pattern, it is necessary to take a careful history taking and perform an additional examination such as CT and MRI for the accurate diagnosis. In this case, patient presented the following atypical pain: First, patient complained spontaneous pain but has no increase in pain during opening and mastication. Second, he showed severe pain in the left TMJ though there was no pain during opening, protrusive and lateral movement. Finally, He took strong nonsteroidal anti-inflammatory drugs but his symptoms persisted. Untreated COM can spread, leading to condylar resorption and SBO. Untreated SBO can lead to death. If COM patient over 60 years of age has a history of systemic diseases, such as diabetes mellitus to have a high risk of spreading the infection, fungal osteomyelitis may develop up to the skull base, leading to condylar bony change. It is important to diagnose early by using CT or MRI and check regularly to prevent the spread of the disease after diagnosis.

\section{CONFLICT OF INTEREST}

No potential conflict of interest relevant to this article was reported.

\section{ORCID}

\author{
Bok Eum Kim \\ https://orcid.org/0000-0001-7396-2479 \\ Keun Jeong Park \\ https://orcid.org/0000-0003-4559-9398 \\ Jung Eun Lee \\ https://orcid.org/0000-0001-6439-8087 \\ YounJung Park \\ https://orcid.org/0000-0002-9152-7849 \\ Jeong-Seung Kwon \\ https://orcid.org/0000-0003-4584-7355 \\ Seong-Taek Kim \\ https://orcid.org/0000-0001-9506-5103 \\ Jong-Hoon Choi \\ https://orcid.org/0000-0003-3211-3619 \\ Hyung-Joon Ahn \\ https://orcid.org/0000-0001-9669-9781
}

\section{REFERENCES}

1. Bhutta MF, Thornton RB, Kirkham LS, Kerschner JE, Cheeseman MT. Understanding the aetiology and resolution of chronic otitis media from animal and human studies. Dis Model Mech 2017;10:1289-1300.

2. Wallis S, Atkinson H, Coatesworth AP. Chronic otitis media. Postgrad Med 2015;127:391-395.

3. Luscan R, Belhous K, Simon F, et al. TMJ arthritis is a frequent complication of otomastoiditis. J Craniomaxillofac Surg 2016;44:1984-1987.

4. Leskinen K, Jero J. Acute complications of otitis media in adults. Clin Otolaryngol 2005;30:511-516.

5. Leichtle A, Hoffmann TK, Wigand MC. [Otitis media: definition, pathogenesis, clinical presentation, diagnosis and therapy]. Laryngorhinootologie 2018;97:497-508. German.

6. Bastianpillai J, Chaudhry S, Vijendren A. Rare case of a 3-yearold with Candida skull base osteomyelitis: lessons to be learnt. BMJ Case Rep 2019;12:e228026.

7. Stodulski D, Kowalska B, Stankiewicz C. Otogenic skull base osteomyelitis caused by invasive fungal infection. Case report and literature review. Eur Arch Otorhinolaryngol 2006;263:1070-1076.

8. Clark MP, Pretorius PM, Byren I, Milford CA. Central or atypical skull base osteomyelitis: diagnosis and treatment. Skull Base 2009;19:247-254.

9. Menachof MR, Jackler RK. Otogenic skull base osteomyelitis caused by invasive fungal infection. Otolaryngol Head Neck Surg 1990;102:285-289.

10. Gamaletsou MN, Kontoyiannis DP, Sipsas NV, et al. Candida osteomyelitis: analysis of 207 pediatric and adult cases (1970-2011). 
Clin Infect Dis 2012;55:1338-1351.

11. Conde-Díaz C, Llenas-García J. Severe skull base osteomyelitis caused by pseudomonas aeruginosa with successful outcome after prolonged outpatient therapy with continuous infusion of ceftazidime and oral ciprofloxacin: a case report. J Med Case Rep 2017;11:48.

12. Miyabe H, Uno A, Nakajima T, et al. A case of skull base osteomyelitis with multiple cerebral infarction. Case Rep Otolaryngol 2016;2016:9252361.
13. Muranjan SN, Khadilkar SV, Wagle SC, et al. Central skull base osteomyelitis: diagnostic dilemmas and management issues. Indian J Otolaryngol Head Neck Surg 2016; 68:149-156.

14. Bariteau JT, Waryasz GR, McDonnell M, Fischer SA, Hayda RA, Born CT. Fungal osteomyelitis and septic arthritis. J Am Acad Orthop Surg 2014;22:390-401.

15. Blyth CC, Gomes L, Sorrell TC, da Cruz M, Sud A, Chen SC. Skullbase osteomyelitis: fungal vs. bacterial infection. Clin Microbiol Infect 2011;17:306-311. 